Draft VERSion June 20, 2018

Preprint typeset using $\mathrm{LAT}_{\mathrm{E}} \mathrm{X}$ style emulateapj v. 12/16/11

\title{
RAPID FORMATION OF SATURN AFTER JUPITER COMPLETION
}

\author{
Hiroshi Kobayashi ${ }^{1,2}$, Chris W. Ormel ${ }^{3,4}$ And Shigeru IdA ${ }^{5}$ \\ Draft version June 20, 2018
}

\begin{abstract}
We have investigated Saturn's core formation at a radial pressure maximum in a protoplanetary disk, which is created by gap opening by Jupiter. A core formed via planetesimal accretion induces the fragmentation of surrounding planetesimals, which generally inhibits further growth of the core by removal of the resulting fragments due to radial drift caused by gas drag. However, the emergence of the pressure maximum halts the drift of the fragments, while their orbital eccentricities and inclinations are efficiently damped by gas drag. As a result, the core of Saturn rapidly grows via accretion of the fragments near the pressure maximum. We have found that in the minimum-mass solar nebula, kilometer sized planetesimals can produce a core exceeding 10 Earth masses within two million years. Since Jupiter may not have undergone significant type II inward migration, it is likely that Jupiter's formation was completed when the local disk mass has already decayed to a value comparable to or less than Jovian mass. The expected rapid growth of Saturn's core on a timescale comparable to or shorter than observationally inferred disk lifetime enables Saturn to acquire the current amount of envelope gas before the disk gas is completely depleted. The high heat energy release rate onto the core surface due to the rapid accretion of the fragments delays onset of runaway gas accretion until the core mass becomes somewhat larger than that of Jupiter, which is consistent with the estimate based on interior modeling. Therefore, the rapid formation of Saturn induced by gap opening of Jupiter can account for the formation of multiple gas giants (Jupiter and Saturn) without significant inward migration and larger core mass of Saturn than that of Jupiter.

Subject headings: planets and satellites:formation — solar system: formation — planets and satellites: individual (Jupiter, Saturn)
\end{abstract}

\section{INTRODUCTION}

Since Jupiter resides at $5.2 \mathrm{AU}$, it is likely that Jupiter did not undergo significant type II migration (e.g., Ida \& Lin 2008). The mass of the disk surrounding Jupiter decayed to a level comparable to or less than Jupiter mass before Jupiter completed its formation and opened up a gap. Since the disk could not push Jupiter, its type II migration is negligible. The formation of Saturn becomes problematic along this line. With an assumption that cores grow through the collisional accretion of surrounding planetesimals, the accretion timescale for Saturn's core may be 5-10 times longer than that for Jupiter's core. Therefore, since Jupiter forms on a timescale comparable to a disk depletion timescale, a few Myrs, Saturn's core formed on a timescale of 10 30 Myrs. However, when the core formed, the disk mass should have been so severely depleted that the core cannot accrete disk gas as massive as the present envelope mass. To reconcile the inconsistency, Saturn's core should have formed on a timescale shorter than several Myrs after Jupiter's formation. Note that a scenario by

hkobayas@nagoya-u.jp

ormel@astro.berkeley.edu

ida@geo.titech.ac.jp

1 Department of Physics, Nagoya University, Nagoya, Aichi 464-8602, Japan

2 Astrophysical Institute and University Observatory, Friedrich Schiller University, Schillergaesschen 2-3, 07745 Jena, Germany

3 Astronomy Department, University of California, Berkeley, CA94720, USA

4 Hubble Fellow.

5 Tokyo Institute of Technology, Ookayama, Meguro-ku, Tokyo 152-8551, Japan
Walsh et al. (2011) where Jupiter first migrates inward, and then outward (e.g., Morbidelli \& Crida 2007), also requires rapid formation of Saturn.

A single planetary embryo is formed from collisional coagulation of planetesimals in an annulus of the disk along the embryo's orbit and further grows through collisions with surrounding remnant planetesimals. An embryo reaches the critical core mass, $\sim 10 M_{\oplus}$, to start gas accretion for Saturn formation (Mizuno 1980; Bodenheimer \& Pollack 1986; Ikoma et al. 2000). However, since massive embryos enlarge the random motion of planetesimals, collisions between planetesimals are destructive. Embryos also grow by the accretion of fragments that result from such collisions. The eccentricities and inclinations of fragments are well damped by gas drag, and fragment accretion accelerates embryo growth. On the other hand, since such small fragments quickly drift inwards, the final mass of an embryo is much smaller than the critical core mass to start gas accretion (Kobavashi et al. 2010, 2011; Ormel \& Kobavashi 2012). However, Jupiter may have opened up a gap in a disk to truncate gas inflow to Jupiter at the present Jupiter's mass, which forms a radial pressure maximum near the edge of the gap. Radial drift of fragments is stalled at around the pressure maximum (Adachi et al. 1976). In addition, type I migration of a core is also stalled there (Tanaka et al. 2002; Masset et al. 2006). Since a core grows via fragment accretion without the loss of fragments and the core itself, rapid formation of Saturn's core may be realized under these conditions.

In order to investigate the rapid formation of Saturn core near the edge of the gap, we perform simulations, 
which can derive accurate solutions in both limits dominated by collisional fragmentation (Kobavashi \& Tanaka 2010) or coagulation (Kobavashi et al.|2010). In Section 2 we investigate the radial drift of bodies near the pressure maximum. We model a disk for a simulation and briefly explain the method of the simulation in Section 3. The core growth derived by the simulations is shown in Section 4. We discuss our findings in Section [5 and present our conclusion in Section 6 .

\section{RADIAL DRIFT NEAR PRESSURE MAXIMA}

The velocity distinction between the gas rotational velocity $v_{\text {gas }}$ and the Keplarian velocity $v_{\mathrm{k}}$ is given by $v_{\mathrm{K}}-v_{\text {gas }}=\eta v_{\mathrm{K}}$ with (Adachi et al. 1976)

$$
\eta=-\frac{1}{2 \rho_{\text {gas }} a \Omega_{\mathrm{K}}^{2}} \frac{d P_{\mathrm{gas}}}{d a},
$$

where $\rho_{\text {gas }}$ and $P_{\text {gas }}$ are, respectively, the gas density and pressure of disk midplane, $\Omega_{\mathrm{K}}$ is the Keplarian angular velocity, and $a$ is the distance from the sun. Equation (10) shows $\eta=0$ at a pressure maximum.

Gap opening by Jupiter changes the radial profile of gas surface density, which determines $\eta$. Tanigawa \& Ikoma (2007) presented an analytical formula for the gas surface density $\Sigma_{\mathrm{g}}$ around the gap a:

$$
\begin{aligned}
\Sigma_{\mathrm{g}} & =\Sigma_{\mathrm{g}, \infty} \exp \left[-\left(a-a_{\mathrm{J}} / l\right)^{-3}\right], \\
l & =\left[\frac{8}{81 \pi} \frac{a_{\mathrm{J}}^{2} \Omega_{\mathrm{K}}\left(a_{\mathrm{J}}\right)}{\nu}\left(\frac{M_{\mathrm{J}}}{M_{\odot}}\right)^{2}\right]^{1 / 3} a_{\mathrm{J}},
\end{aligned}
$$

where $a_{\mathrm{J}}$ is the orbital radius of Jupiter, the viscosity coefficient $\nu$ is given by $\alpha c^{2} \Omega_{\mathrm{K}}^{-1}$ with the sound velocity $c$ and a parameter $\alpha, \Sigma_{\mathrm{g}, \infty}$ is the gas surface density far away from Jupiter, and $M_{\mathrm{J}}$ and $M_{\odot}$ are, respectively, the masses of Jupiter and the sun. We assume $\Sigma_{\mathrm{g}, \infty} \propto a^{-p}$ and $c \propto a^{-q}$. Equations (11) and (2) then give $\eta$ near the pressure maximum formed by Jupiter as

$$
\eta=\frac{c^{2}}{2 a^{2} \Omega_{\mathrm{K}}^{2}}\left[p+q+\frac{3}{2}-\frac{3 l^{3} a}{\left(a-a_{\mathrm{J}}\right)^{4}}\right] .
$$

Note that Equation (2) gives the density profile determined by viscous torques. The thermal instability of the density profile (Rayleigh criterion) regulates the density profile in the vicinity of Jupiter (Ida \& Lin 2004; Tanigawa \& Ikoma 2007; Crida et al. 2006). However, for the purpose of finding the location of the pressure maximum, we can use Equation (2) as long as $c / \Omega_{\mathrm{K}} \lesssim$ $0.1 a_{\mathrm{J}}$ and $\alpha \lesssim 0.02$.

Bodies lose their angular momenta through gas drag and then drift inward, resulting in their radial transport. The gas drag force acting on a body with radius $r$ and mass $m$ for the relative velocity $u$ between the body and gas is expressed by $C_{\mathrm{D}} \pi r^{2} \rho_{\text {gas }} u^{2} / 2$ with the dimensionless gas drag coefficient $C_{\mathrm{D}}$, which is determined taking into account Epstein and Stokes regimes as well as constant $C_{\mathrm{D}}$ for high Reynolds number. The stopping time

\footnotetext{
6 Crida et al. (2006) derived a similar formula for the density profile of gap. The locations of the pressure maximum (8.9 AU for $\alpha=6.9 \times 10^{-5} ; 7.2 \mathrm{AU}$ for $\alpha=10^{-3}$, where we adopt $c / \Omega_{\mathrm{K}} a \approx 0.05$ at $a=a_{\mathrm{J}}$ ) are almost the same as the values derived from the formula by Tanigawa \& Ikoma (2007).
}

normalised by $\Omega_{\mathrm{K}}^{-1}$ is given by

$$
\tilde{\tau}_{\text {stop }}=a \Omega_{\mathrm{K}}^{2} \tau / u,
$$

where

$$
\tau=\frac{2 m}{\pi r^{2} C_{\mathrm{D}} \rho_{\text {gas }} v_{\mathrm{K}}} .
$$

We do not consider very small fragments of $\tilde{\tau}_{\text {stop }} \ll 1$ because radial drift of these fragments is negligible due to strong coupling with gas. The relative velocity $u$ then depends on eccentricity and inclination of a body as well as on $\eta$. Eccentricities and inclinations of bodies have the Rayleigh-type distributions with dispersions $e^{*}$ and $i^{*}$, respectively (Ida \& Makino 1992). The averaged value of $u$ is estimated as $\left(e^{*}+i^{*}+\eta\right) a \Omega_{\mathrm{K}}$. The drift velocity $v_{\mathrm{d}}$ is analytically given in the limit of $\tilde{\tau}_{\text {stop }} \lesssim 1$ or $\tilde{\tau}_{\text {stop }} \gg 1$ (Adachi et al. 1976). Averaging the solutions for bodies with eccentricities and inclinations in Rayleigh-type distribution and connecting both these limits, we then have 7

$$
\begin{aligned}
v_{\mathrm{d}}= & -2 \frac{a}{\tau} \frac{\tilde{\tau}_{\text {stop }}^{2}}{1+\tilde{\tau}_{\text {stop }}^{2}}\left[\eta\left(3.0 e^{* 2}+1.3 i^{* 2}+\eta^{2}\right)^{1 / 2}\right. \\
& \left.+3.5 e^{* 3}+0.42 i^{* 3}\right] .
\end{aligned}
$$

Equation (7) covers both limits in $\tilde{\tau}_{\text {stop }} \lesssim 1$ and $\tilde{\tau}_{\text {stop }} \gg$ 1. Although the drift velocity becomes very low at the pressure maximum because of $\eta=0$, bodies with high $e^{*}$ or $i^{*}$ can drift due to the cubic terms of $e^{*}$ and $i^{*}$.

A massive embryo produced in an annulus of the disk stirs surrounding smaller bodies. The stirring and damping due to gas drag determine $e^{*}$ and $i^{*}$ of bodies, depending on their radii. Since $e^{*}$ and $i^{*}$ increase with embryo growth, bodies drift inward from the pressure maximum. However, since $\eta$ is negative inside the pressure maximum, the inward drift due to $e^{*}$ and $i^{*}$ is canceled out at a certain distance by the outward one caused by negative $\eta$. For $\alpha=6.9 \times 10^{-5}, p=3 / 2$, and $q=1 / 4$, the radial distribution of $\Sigma_{\mathrm{g}}$ and $\eta$ is shown in Fig. 10 8 ; the pressure maximum is then located at $9 \mathrm{AU}$. Fig. 1 also shows the location of $v_{\mathrm{d}}=0$ as a function of the radius of bodies. The location of $v_{\mathrm{d}}=0$ for small bodies is close to $9 \mathrm{AU}$, because $e^{*}$ and $i^{*}$ are strongly damped by gas drag. Although the location of $v_{\mathrm{d}}=0$ moves inward for a massive embryo because of its strong stirring, the feeding zone of the embryo, of which the width is given by 10 times Hill radii of the embryo (Kokubo \& Ida 1998), is so wide that the embryo can accrete kilometer-sized or smaller bodies. Although larger bodies drift out of the feeding zone, the drift timescale is much longer than the timescale of collisional fragmentation among them or of the accretion onto the embryo. Note that even if such large bodies drift out of the feeding zone, small bodies resulting from collisional fragmentation of the large bodies drift outward and halt inside the feeding zone. Therefore, the assumption of $v_{\mathrm{d}} \approx 0$ is valid to investigate embryo grow at the pressure maximum.

\footnotetext{
7 Inaba et al. (2001) modified terms related to $\eta$ in the analytical formula for $\tilde{\tau}_{\text {stop }} \gg 1$. We apply the modified formula for terms related to $\eta$ but the cubic terms of $e^{*}$ and $i^{*}$ from Adachi et al. $(1976)$.

${ }^{8}$ We discuss the applied $\alpha$ value below.
} 


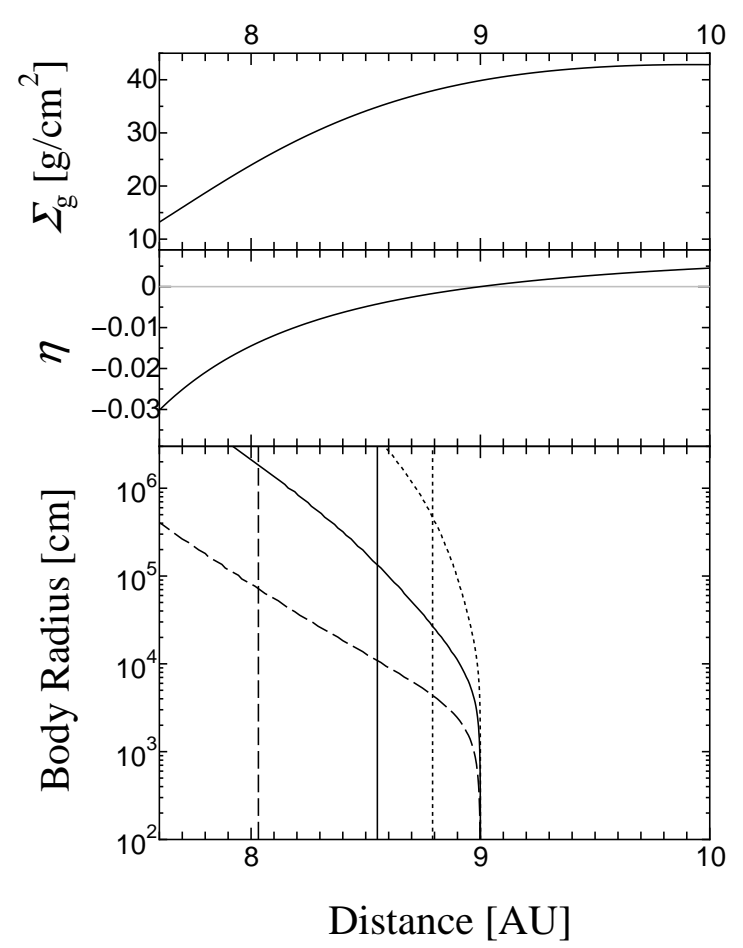

FIG. 1. - The radial distribution of the surface density $\Sigma_{\mathrm{g}}$ (top) and $\eta$ given by Equation (4) (middle) and the location of $v_{\mathrm{d}}=0 \mathrm{vs}$. the radius of bodies (bottom). In order to determine the location, eccentricities of the bodies are given by the gas drag damping and the stirring of an embryo with 0.1 (dotted lines), 1 (solid line), 10 (dashed lines) Earth masses. The vertical lines indicate the inner edge of the feeding zone of the embryo residing at $9 \mathrm{AU}$.

The location of the pressure maximum depends on the turbulent strength, $\alpha$ value. Magneto-rotational instability may cause turbulence in the disk; its activity depends on an ionization degree and the strength of magnetic field. Because of the low ionization degree, a magnetically decoupled midplane dead zone emerges at $\lesssim 20 \mathrm{AU}$ (Sano et al. 2000) 9 . In the dead zone, the $\alpha$ value is expected to be as low as $10^{-5}-10^{-4}$ if the net vertical magnetic field is weak enough (Okuzumi \& Hirose 2011; Gressel et al. 2012); the pressure maximum is then located at around $9 \mathrm{AU}$. For larger $\alpha \approx 10^{-3}$, the pressure maximum moves to about $7 \mathrm{AU}$. However, the behavior of bodies is similar; bodies smaller than $1-10 \mathrm{~km}$ halt inside the feeding zone of an embryo formed at the pressure maximum and thereby these bodies contribute to the embryo growth. Therefore, the location of pressure maximum (or choosing $\alpha$ value) insignificantly affects the Saturn core formation. We thus fix the pressure maximum at $9 \mathrm{AU}$ in the following.

\section{MODEL}

We introduce a disk model for the initial surface mass density of solids $\Sigma_{\mathrm{s}, 0}$ and gas $\Sigma_{\mathrm{g}, 0}$ such that

$$
\Sigma_{\mathrm{s}, 0}=x_{\mathrm{s}} \Sigma_{\mathrm{MMSN}, \mathrm{s}}\left(\frac{a}{9 \mathrm{AU}}\right)^{-3 / 2} \mathrm{~g} \mathrm{~cm}^{-2},
$$

${ }^{9}$ Although a reduction of the vertical optical depth of the disk due to dust depletion could make the dead zone small (Sano et al. 2000), in our model very small bodies supplied by collisional fragmentation, whose total mass is negligible as shown in Section 4 can produce a sufficient optical depth.

$$
\Sigma_{\text {gas }, 0}=x_{\mathrm{g}} \Sigma_{\mathrm{MMSN}, \mathrm{g}}\left(\frac{a}{9 \mathrm{AU}}\right)^{-3 / 2} \mathrm{~g} \mathrm{~cm}^{-2}
$$

where $\Sigma_{\mathrm{MMSN}, \mathrm{s}}=1.1 \mathrm{~g} \mathrm{~cm}^{-2}$ and $\Sigma_{\mathrm{MMSN}, \mathrm{g}}=89 \mathrm{~g} \mathrm{~cm}^{-2}$ are, respectively, the solid and gas surface densities at $9 \mathrm{AU}$ in the minimum-mass solar nebula (MMSN) model (Havashi 1981). We vary scaling factors $x_{\mathrm{s}}$ and $x_{\mathrm{g}}$ to find the conditions for Saturn core formation. Solid surface density evolves in the simulation but gas density is set to be constant with time. We consider the disks with $x_{\mathrm{g}} \sim 1$ which are small enough for Jupiter to reside at the current position without significant type II migration and large enough to form cores for Jupiter and Saturn (Ida \& Lin 2008).

We consider a disk from 8.6 AU to $35 \mathrm{AU}$. The disk is divided into 14 annuli whose widths are given by 0.1 times the distance from the annulus center to the sun. The innermost annulus centered at $9 \mathrm{AU}$ is assumed to be the pressure maximum zone. As shown in Section 2. kilometer-sized or smaller bodies stay in the feeding zone of an embryo residing at the pressure maximum. In the MMSN disk, drift time required for larger bodies to go out of the feeding zone of an embryo with mass $\leq 10 M_{\oplus}$ is longer than the formation timescale of Saturn's core, $1-2 \times 10^{6}$ years, if the bodies are larger than about $30 \mathrm{~km}$. For bodies with intermediate sizes, the drift time is longer than their collisional fragmentation timescale (Kobavashi \& Tanaka 2010). Therefore, we ignore radial drift of bodies in the innermost annulus $\left(v_{\mathrm{d}}=0\right)$. The values of $\Sigma_{\mathrm{g}, 0}$ and $\eta$ are taken from those at the radial center of each annulus, given by Equation (91) and $\eta=5.4 \times 10^{-3}(a / 9 \mathrm{AU})^{1 / 2}$ except for the innermost annulus that has $\eta=0$. Each annulus has a lot of mass bins to calculate the evolution of the mass distribution of bodies via collisional coagulation and fragmentation among bodies. Collisional outcomes are assumed to be scaled by the dimensionless impact energy $\phi=m_{1} m_{2} v^{2} / 2\left(m_{1}+m_{2}\right)^{2} Q_{\mathrm{D}}^{*}$ according to the simple model that Kobavashi \& Tanaka (2010) and Kobayashi et al. (2010) constructed consistently with laboratory experiments and collisional simulations, where $m_{1}$ and $m_{2}$ are the collider masses, $v$ is the collisional velocity between the colliders and $Q_{\mathrm{D}}^{*}$ is the specific energy needed to eject half of the total mass of the colliders. The collisional cross sections of bodies depend on their random velocities determined by $e^{*}$ and $i^{*}$. Since the stirring of large bodies increases the velocities, the mass distribution of bodies affects the velocity evolution. Therefore, we calculate the mass and velocity evolution simultaneously using the collision rates (Inaba et al. 2001) and the velocity evolution rates due to mutual interaction of bodies (Ohtsuki et al. 2002) and due to gas drag (Adachi et al. 1976). With a use of an upwind scheme, we calculate radial transport of bodies due to radial drift given by Equation (7) except for the innermost annulus.

In addition, we include the enhancement of collisions with embryos due to their tenuous atmospheres and due to gas drag for small bodies with $\tilde{\tau}_{\text {stop }} \sim 1$, where $\tilde{\tau}_{\text {stop }}=1$ corresponds to several tens centimeter in radius at $9 \mathrm{AU}$. We apply the atmospheric enhancement modeled by Inaba \& Ikoma (2003) with the grain depletion factor $f=1 \times 10^{-2}$ and the collision rate between embryos and small bodies with $\tilde{\tau}_{\text {stop }} \sim 1$, given by 
Ormel \& Klahr (2010). For the Ormel \& Klahr model, we adopt the headwind velocity of gas to an embryo as $\left(\eta+e_{\mathrm{E}}+i_{\mathrm{E}}\right) v_{\mathrm{K}}$, where $e_{\mathrm{E}}$ and $i_{\mathrm{E}}$ are, respectively, eccentricity and inclination of the embryo.

Since small bodies are strongly coupled with gas flow, the model of Ormel \& Klahr gives a very long timescale for collisions between very small bodies and embryos. In addition, because the collisional cascade caused by embryo growth stalls at a body size of about $1-10 \mathrm{~m}$, the total mass of smaller bodies is negligible (Kobavashi et al. 2010). Therefore, we neglect bodies with radius smaller than $r_{\min }$, where $r_{\min }=0.01 \mathrm{~cm}$ in our simulation. Indeed, embryo growth is independent of $r_{\min }$ if $r_{\min } \ll$ $1 \mathrm{~cm}$.

\section{SATURN CORE FORMATION}

We perform simulations for core formation and growth starting from a monodisperse mass population of planetesimals of mass $m_{0}$ and radius $r_{0}$ with $e^{*}=2 i^{*}=$ $\left(2 m_{0} / 3 M_{\odot}\right)^{1 / 3}$. In Fig. 2, the evolution of the size distribution due to collisions is shown for $r_{0}=1 \mathrm{~km}$ $\left(m_{0}=4.2 \times 10^{15} \mathrm{~g}\right)$ in the MMSN model $\left(x_{\mathrm{s}}=x_{\mathrm{g}}=1\right)$. Collisional coagulation of planetesimals produces a small number of large bodies, planetary embryos. As embryos grow via the accretion of a swarm of planetesimals, massive embryos stir planetesimals and collisional fragmentation among planetesimals turns planetesimals into small fragments. Since gas drag suppresses the random motions of very small bodies, fragmentation by collisions among such small bodies no longer occurs. The grinding down of bodies through collisions halts at $\sim 10 \mathrm{~m}$, where bodies accumulate (see Fig. 21). The mass distribution is given by three discrete components that are the largest bodies (planetary embryos), initial sized or slightly larger bodies (planetesimals), and small bodies with 1-100 m.

The gas drag coefficient $C_{\mathrm{D}}$ is constant for kilometersized or larger bodies but it increases with decreasing size in the Stokes regime, given by $C_{\mathrm{D}}=5.5 \mathrm{cl} / \mathrm{g} / \mathrm{ur}$ (Adachi et al. 1976). Here, $l_{\mathrm{g}}=l_{\mathrm{g}, 0} / \rho_{\text {gas }}$ is the mean free path of gas molecular with $l_{\mathrm{g}, 0}=1.7 \times 10^{-9} \mathrm{~g} \mathrm{~cm}^{-2}$. The fragments accumulating at $1-100 \mathrm{~m}$ are controlled by the Stokes drag. The equilibrium eccentricity $e_{\mathrm{eq}}^{*}$ in the Stokes regime is estimated as (Kobayashi et al. 2010, 2011)

$$
e_{\mathrm{eq}}^{* 2}=\frac{h_{M}^{3}\left\langle P_{\mathrm{VS}}\right\rangle \tau a \Omega_{\mathrm{K}}^{2}}{2^{7 / 3} \pi \tilde{b} u},
$$

where $h_{M}=\left(M / 3 M_{\odot}\right)^{1 / 3}$ is the reduced Hill radius of an embryo with mass $M,\left\langle P_{\mathrm{VS}}\right\rangle=73$ is the dimensionless stirring rate for $e^{*} \ll h_{M}$, and $\tilde{b}=10$ is the separation of embryos divided by their mutual Hill radius. Since $\tau \propto u$ in the Stokes regime, $e_{\mathrm{eq}}^{*}$ is independent of $u$; the eccentricity is the same as that in the case $\eta \neq 0$. When a body of mass $m$ collides with a similar-sized body, the impact energy is given by $m\left(e_{\mathrm{eq}}^{*} v_{\mathrm{k}}\right)^{2} / 4$. If the energy is much smaller than $2 m Q_{\mathrm{D}}^{*}$, the collision no longer produces significant fragments. The collisional cascade stalls around $\left(e_{\mathrm{eq}}^{*} v_{\mathrm{k}}\right)^{2}=C_{\mathrm{L}} Q_{\mathrm{D}}^{*}$ with a constant $C_{\mathrm{L}} \sim 1$ and small bodies accumulate around the radius satisfying the condition, which is obtained from the condition and Equation (10) as

$$
r_{\mathrm{f}}=7.9 C_{\mathrm{L}}^{1 / 2}\left(\frac{Q_{\mathrm{D}}^{*}}{8.8 \times 10^{6} \mathrm{erg} \mathrm{g}^{-1}}\right)^{1 / 2}
$$

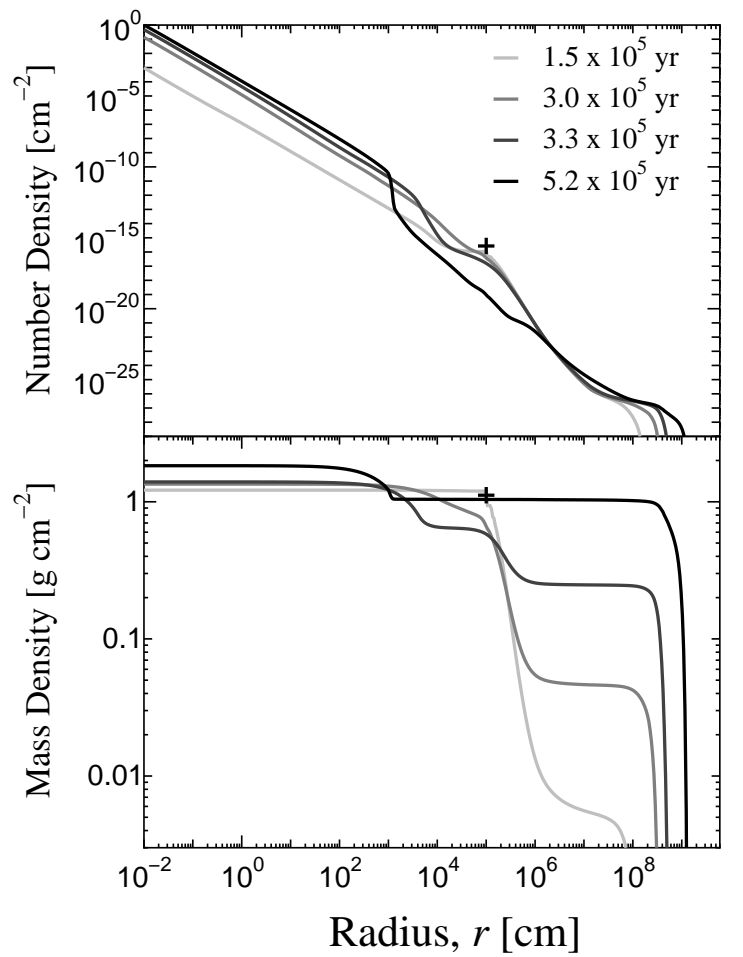

FIG. 2.- Evolution of surface number and mass densities of bodies larger than radius $r$ at $9 \mathrm{AU}$, starting from a swarm of planetesimals with initial radius $r_{0}=1 \mathrm{~km}$ in the MMSN model. Crosses indicate the initial condition and lines are size distributions at different times.

$$
\times\left(\frac{T}{93 \mathrm{~K}}\right)^{1 / 4}\left(\frac{a}{9 \mathrm{AU}}\right)^{5 / 4}\left(\frac{M}{M_{\oplus}}\right)^{-1 / 2} \mathrm{~m},
$$

where $T$ is the disk midplane temperature and the value of $Q_{\mathrm{D}}^{*}$ for a body of radius $r=1 \mathrm{~m}$ is applied (Benz \& Asphaug 1999). Indeed, $r_{\mathrm{f}}$ is consistent with the result of simulations for $C_{\mathrm{L}} \sim 1$ (see Fig. 21). Interestingly, $r_{\mathrm{f}}$ is independent of $\eta$ and $\rho_{\text {gas }}$ because gas drag is determined in the Stokes regime. In addition, $e_{\mathrm{f}}^{*}=\left(C_{\mathrm{L}} Q_{\mathrm{D}}^{*}\right)^{1 / 2} / v_{\mathrm{K}} \approx 3 \times 10^{-3}(a / 9 \mathrm{AU})^{1 / 2}$ is smaller than $h_{M}=10^{-2}\left(M / M_{\oplus}\right)^{1 / 3}$, resulting in a very large accretion rate of the fragments onto an embryo (Ida \& Nakazawa 1989).

Fig. 3 shows the evolution of embryo masses 10 at $9 \mathrm{AU}$ for the MMSN disk with $r_{0}=1 \mathrm{~km}$ and $10 \mathrm{~km}$. Embryos initially grow exponentially with time and the growth time scale is proportional to $r_{0} / \Sigma_{\mathrm{s}, 0}$ (Ormel et al. 2010a, b), which is called runaway growth. Massive embryos formed by the runaway growth stir remnant planetesimals and then slower, oligarchic growth starts for embryos larger than $\sim 10^{-3} M_{\oplus}$. Such massive embryos then activate collisional fragmentation of planetesimals. The very low $e^{*}$ and $i^{*}$ of the small resulting fragments damped by strong gas drag tend to accelerate the embryo growth. On the other hand, the gas drag also leads to the radial drift of the fragments, which generally limits the embryo growth (Kobavashi et al. 2010, 2011). How-

10 In our simulation, we define "runaway bodies" which cannot collide with each other due to their large orbital separations (see Kobavashi et al. 2010). Here "embryo masses" represent the averaged mass of runaway bodies, which roughly corresponds to the mass of the largest body. 
ever, near the pressure maximum due to gap opening by Jupiter, embryos effectively accrete such fragments without the loss of the fragments. In Fig. 3, the embryo growth starts being accelerated at $\sim 0.01 M_{\oplus}$ (at about $3 \times 10^{5}$ years for $r_{0}=1 \mathrm{~km}$ and at about $3 \times 10^{6}$ years for $r_{0}=10 \mathrm{~km}$ ), because fragment production becomes efficient at such an embryo mass. At $\gtrsim M_{\oplus}$, the acceleration slows down due to depletion of the total amount of surrounding bodies by the embryo accretion. Nevertheless, the embryo keeps growing beyond the "isolation mass", which is several Earth masses for the MMSN model, because fragments produced in outer annuli drift into the region at $\sim 9 \mathrm{AU}$. Eventually, an embryo reaches 10 Earth masses in $2 \times 10^{6}$ years for $r_{0}=1 \mathrm{~km}$, while the embryo mass is about an Earth mass in $5 \times 10^{6}$ years for $r_{0}=10 \mathrm{~km}$.

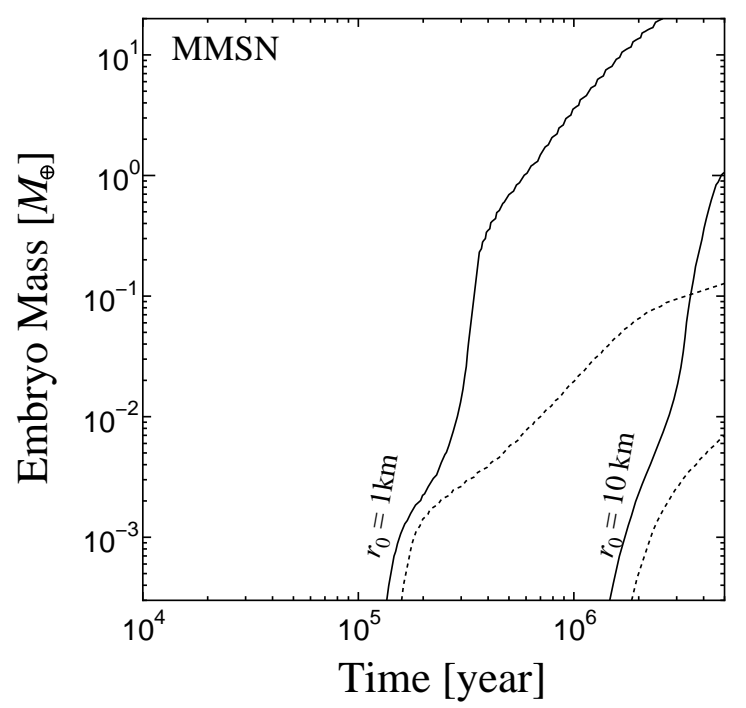

FIG. 3.- Embryo growth at $9 \mathrm{AU}$ for $r_{0}=1 \mathrm{~km}$ and $10 \mathrm{~km}$ in the MMSN model $\left(x_{\mathrm{g}}=x_{\mathrm{s}}=1\right)$. For reference, dotted lines show the results in the case of an unperturbed disk (i.e. without Jupiter's gap).

In the oligarchic growth of embryos, embryos have orbital separations of 10 Hill radii of themselves (Kokubo \& Ida 1998). Since 10 Hill radii of an embryo with $10 M_{\oplus}$ at $9 \mathrm{AU}$, about $2 \mathrm{AU}$, is larger than the pressure maximum region (see Fig. 1), only an embryo larger than $10 M_{\oplus}$ can be formed in the pressure maximum. In case that the pressure maximum does not emerge (dotted lines in Fig. 3), embryo growth is not so rapid and stalls at about $0.1 M_{\oplus}$, because surrounding planetesimals are lost due to combination of planetesimal fragmentation with the radial drift of resulting fragments (see also Kobayashi et al. 2010, 2011). Since there is no pressure maximum in the outer disk, embryos beyond 10 AU cannot become larger than a body of about Mars mass. Meanwhile the fragmentation of planetesimals and the ensuing radial drift of resultant fragments remove the solid mass reservoir in the outer disk, which facilitates the formation of a large enough embryo in the pressuremaximum region.

Although a core can keep growing at the pressure maximum, the core is required to reach $\sim 10 M_{\oplus}$ within several Myrs for the formation of Saturn. For $r_{0} \gtrsim 10 \mathrm{~km}$, an MMSN disk $\left(x_{\mathrm{s}}=x_{\mathrm{g}}=1\right)$ cannot produce a core with 10 Earth masses in several million years (see Fig. 3). Fragment production from large planetesimals is ineffective because of large $Q_{\mathrm{D}}^{*}$ and occasional collisions among them, which delays embryo growth via fragment accretion. A large solid surface density $\left(x_{\mathrm{s}}>1\right)$ that increases fragment production is necessary for planetesimals with larger $r_{0}$ to form a massive core in several million years. A core can reach 10 Earth masses in a disk with $x_{\mathrm{s}} \gtrsim 3$ for $r_{0}=10 \mathrm{~km}$ and with $x_{\mathrm{s}} \gtrsim 10$ for $r_{0}=100 \mathrm{~km}$ (see Fig. (4). Note that the growth of cores is almost independent of $x_{\mathrm{g}}$, because fragments with $r_{\mathrm{f}}$ contributing most to the growth are controlled by Stokes drag (e.g., Beauge et al. (1994).

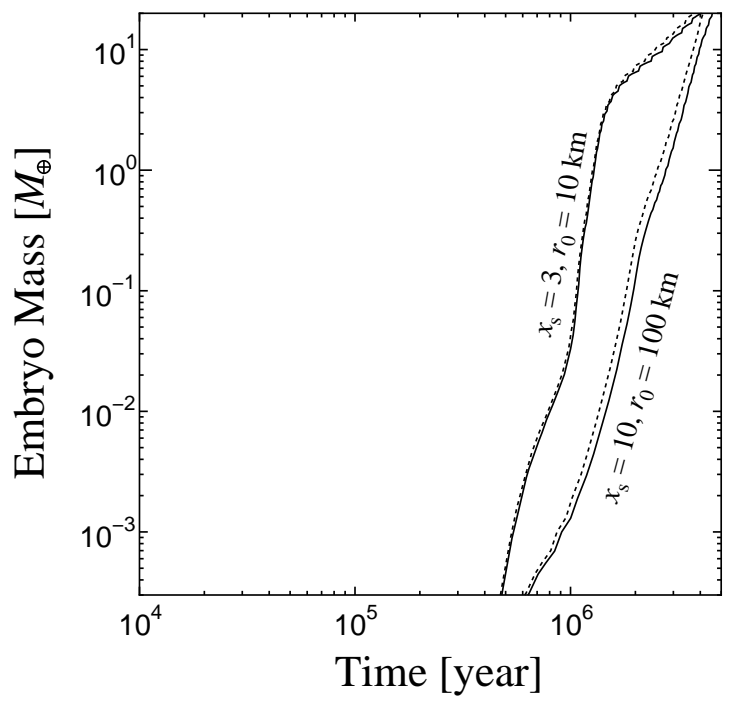

FIG. 4. - Embryos can reach 10 Earth masses at $9 \mathrm{AU}$ in a massive disk with $x_{\mathrm{s}}=3$ for $r_{0}=10 \mathrm{~km}$ and with $x_{\mathrm{s}}=10$ for $r_{0}=100 \mathrm{~km}$. The gas density of the disk is determined as $x_{\mathrm{g}}=1$ (solid lines) and $x_{\mathrm{g}}=x_{\mathrm{s}}$ (dotted lines).

\section{DISCUSSION}

In this paper, we have taken the size of initial planetesimals as a free parameter. The size depends on the formation mechanisms of planetesimals. If planetesimals are formed via self-gravitational instability in a thin dust layer (Goldreich \& Ward 1973; Michikoshi et al. 2012; Takeuchi \& Ida 2012) or of dust accumulated in the structure of disk turbulence (Johansen et al. 2007; Cuzzi et al. 2008), resulting planetesimals are $10 \mathrm{~km}$ or larger. On the other hand, collisional coagulation of fluffy dust is possible to produce planetesimals overcoming an obstacle of rapid radial drift at $\tilde{\tau}_{\text {stop }} \sim 1$ (Okuzumi et al. 2012); the resulting planetesimals may be smaller. In addition, bodies in a significant amount drift from the outer disk during the collisional growth of fluffy dust and such drifting dust increases the solid surface density form MMSN to about $4 \times$ MMSN inside $\sim 10 \mathrm{AU}$ (Okuzumi et al. 2012). Therefore, we should address the history of Saturn core formation including planetesimal formation.

A planetary embryo born at the pressure maximum migrates due to interaction with the surrounding gas disk (type I). The time required to go through half the width of the embryo's feeding zone due to type I migration is longer than the growth time of the embryo unless the 
embryo exceeds $0.4 M_{\oplus}$ (Tanaka et al. 2002). The type I migration rate of an embryo with mass $M$ at distance $a$ is given by $d a / d t=2 f_{\mathrm{I}} M \Sigma_{\mathrm{g}} a^{5} \Omega_{\mathrm{K}}^{3} / M_{\odot}^{2} c^{2}$, where $f_{\mathrm{I}}$ is a factor that is determined by $p=-d \ln \Sigma_{\mathrm{g}} / d \ln a$ and $q=-d \ln c / d \ln a$. If horseshoe torques are fully saturated, $f_{\mathrm{I}}=-1.45-0.6 p-1.8 q$ in a locally isothermal disk and $f_{\mathrm{I}}=-1.04-0.77 p-0.77 q$ in an adiabatic disk (Paardekooper et al. 2011). In the outer disk where we assume $p=1.5$ and $q=0.25$, type I migration is inward due to a negative value of $f_{\mathrm{I}}$, while outward migration occurs around the gap opened by Jupiter where the radial $\Sigma_{\mathrm{g}}$ slope has a large positive value. Therefore type I migration stalls at a certain distance under the density profile shown in Fig. 1 the location of zero migration is at 8.6 AU in the locally isothermal disk and at 9.1 AU in the adiabatic disk. Since the location is near the pressure maximum, such a large embryo, which has a wide feeding zone, can grow via the accretion of bodies accumulating at around the pressure maximum. Note that if the torques are fully unsaturated, $f_{\mathrm{I}}=-0.85-p+1.8 q$ in the locally isothermal disk and $f_{\mathrm{I}}=-0.61-2.33 p+2.82 q$ in the adiabatic disk (Paardekooper et al. 2010). Then, $f_{\mathrm{I}}$ is positive (outward migration) if $q$ has a large value. In the optically thick regions (inner disks or massive disks) where viscous heating is more dominant than irradiation, $q$ has a relatively large value. However, in the outer regions $(\sim 9 \mathrm{AU})$ of moderate disks with $x_{\mathrm{g}} \sim 1$ that we are concerned with, it is likely that the disk temperature is determined by irradiation rather than viscous heating and that $q$ has a relatively small value $(\sim 0.25)$. Then, for $p=1-1.5$, type I migration is inward $\left(f_{\mathrm{I}}<0\right)$ and the argument of the migration trap is still applicable. Furthermore, the torque saturation is likely in the disk of interest. The saturation is prevented only if the horseshoe liberation time is comparable to the diffusion/thermal diffusion timescale across the horseshoe width (Paardekooper et al. 2011) and if an embryo has a low enough eccentricity (Bitsch \& Kley 2010). The embryos resulting form our simulations have eccentricities larger than 0.01. Therefore the condition for type I migration in the outer disk where Saturn's core forms is most likely to result in inward migration, except in the vicinity of the gap. Consequently, type I migration does not inhibit the fast formation of Saturn's core but rather strengthens it.

Once a core exceeds a critical core mass, the core has no longer a static atmosphere and rapid gas accretion starts to form a gas giant. Interior modeling of Jupiter and Saturn suggests that Jupiter's core mass is smaller than $10 M_{\oplus}$, while Saturn's core is larger than $10 M_{\oplus}$ (Guillot 2005). Since heating by the accretion of small bodies onto a core stabilises the atmosphere, the critical core mass becomes large for a fast growth core (e.g., Ikoma et al. 2000). Traditional scenarios, in which the core growth is 5-10 times slower for Saturn than for Jupiter, cannot explain the estimate of core masses of Jupiter and Saturn. However, Saturn core grows rapidly at the pressure maximum caused by Jupiter's gap opening. Since the growth is possible to be faster for Saturn than for Jupiter, the resulting core masses of Jupiter and Saturn may be consistent with those derived form the interior modeling.

Since the core of Saturn is estimated to be larger than
$10 M_{\oplus}$ (Guillot 2005), Saturn should be formed via core accretion. We have shown that if Jupiter has already been completed and opened a gap in the disk, the massive core of Saturn has rapidly formed from kilometer sized planetesimals in an MMSN disk or from larger planetesimals in a disk with a larger amount of planetesimals. On the other hand, since small initial planetesimals are quickly removed by combination of fragmentation with gas drag, the core of Jupiter is likely to have formed from large initial planetesimals with $\sim 100 \mathrm{~km}$ at around $5 \mathrm{AU}$ in a $10 \times$ MMSN disk (Kobayashi et al. 2011). Jupiter is possible to form earlier than Saturn core formation, if either (1) the solar nebula is as massive as self-gravitational instability occurs, (2) pressure maxima originally exist in the solar nebula, or (3) planetesimals of size $100 \mathrm{~km}$ are born around $5 \mathrm{AU}$ in a massive disk. All the cases are consistent with the sequential formation of Saturn.

After the gas accretion, Saturn might open up another gap in the disk, resulting in the emergence of an additional pressure maximum. Neptune and Uranus mainly consist of ice with more than $10 M_{\oplus}$ covered with tenuous atmospheres. Near the pressure maximum, such massive icy planets can be formed. Fragments produced in the outer disk accumulate within about the width of the disk scale height near the pressure maximum but the feeding zone of such a massive planet is wider than the scale height; hence only one planet can be produced. Meanwhile, the orbital eccentricity distribution of Kuiper belt objects in the 3:2 mean-motion resonance with Neptune suggests that Neptune migrated outward from around $20 \mathrm{AU}$ to the current position (Malhotra 1995). Neptune was therefore expected to have formed at the pressure maximum and to have then migrated outward due to interaction with the outer planetesimal disk (e.g., Ida et al. 2000; Kirsh et al. 2009). Note that torques from interaction with gas that cause type I migration suppress the planetesimal-driven migration. Since Saturn should form in gas with a high density, high gas-to-solid surface densities render type I migration stronger compared to the planetesimal-driven migration. Therefore, the planetesimal-driven migration is negligible for the Saturn formation. However, Neptune plausibly migrated outward through interactions with planetesimals because of a low gas density. Uranus might then have formed at around the pressure maximum caused by Saturn, where fragments produced from remnant planetesimals that induce the Neptune migration accumulate. Therefore, we should also address Uranus and Neptune formation after Saturn's formation.

\section{CONCLUSION}

We have investigated the core formation of Saturn at the pressure maximum caused by Jupiter's gap opening in the solar nebula. Small particles feel strong gas drag and drift radially. The loss of bodies due to the drift stalls embryo growth before embryos reach the critical core mass (Kobayashi et al. 2010, 2011). Although bodies stirred by a large embryo can drift from the pressure maximum, the drift halts near the pressure maximum due to positive radial slope of gas pressure; hence an embryo at the pressure maximum effectively grows without loss of surrounding bodies (see Section 2). Starting from monodisperse planetesimals, planetary embryos are generated via collisional evolution and stir remnant planetes- 
imals, resulting in fragmentation of planetesimals. Fragments collide with each other and they accumulate at a radius $r_{\mathrm{f}}$, given by Equation (11). The random velocity of bodies with $r_{\mathrm{f}}$ is well damped by gas drag; thereby embryos rapidly accrete such bodies. Fragments produced in the outer disk move to the pressure maximum, which contribute to further embryo growth. Due to the rapid accretion, a core as massive as 10 Earth masses forms in several million years, for kilometer-sized initial planetesimals in an MMSN disk, while the core formation needs a disk with 3 times larger solid surface density for $10 \mathrm{~km}$ planetesimals and with 10 times solid surface density for $100 \mathrm{~km}$ planetesimals. The growth is almost independent of gas density. Since the rapid formation in a disk with moderate mass is consistent with insignificant type II migration of Jupiter and larger core of Saturn than that of Jupiter, Saturn's core may have formed in the pressure maximum after Jupiter opened up a gap in the solar nebula.

We thank S. Inutsuka for his comments and encouragement and H. Tanaka and S. Okuzumi for useful discussion. We also acknowledge D. Lin and E. Kokubo for inspiring us to consider the calculations of induced formation model of Saturn. For C.W.O. support for this work was provided by NASA through Hubble Fellowship grant \#HST-HF-51294.01-A awarded by the Space Telescope Science Institute, which is operated by the Association of Universities for Research in Astronomy, Inc., for NASA, under contract NAS 5-26555.
Adachi, I., Hayashi, C., \& Nakazawa, K. 1976, Progress of Theoretical Physics, 56, 1756

Beauge, C., Aarseth, S. J., \& Ferraz-Mello, S. 1994, MNRAS, 270,21

Benz, W., \& Asphaug, E. 1999, Icarus, 142, 5

Bitsch, B., \& Kley, W. 2010, A\&A, 523, A30

Bodenheimer, P., \& Pollack, J. B. 1986, Icarus, 67, 391

Crida, A., Morbidelli, A., \& Masset, F. 2006, Icarus, 181, 587

Cuzzi, J. N., Hogan, R. C., \& Shariff, K. 2008, ApJ, 687, 1432

Goldreich, P., \& Ward, W. R. 1973, ApJ, 183, 1051

Gressel, O., Nelson, R. P., \& Turner, N. J. 2012, MNRAS, 2714

Guillot, T. 2005, Annual Review of Earth and Planetary Sciences, 33,493

Hayashi, C. 1981, Progress of Theoretical Physics Supplement, 70,35

Ida, S., Bryden, G., Lin, D. N. C., \& Tanaka, H. 2000, ApJ, 534, 428

Ida, S., \& Lin, D. N. C. 2004, ApJ, 604, 388

Ida, S., \& Lin, D. N. C. 2008, ApJ, 673, 487

Ida, S., \& Makino, J. 1992, Icarus, 96, 107

Ida, S., \& Nakazawa, K. 1989, A\&A, 224, 303

Ikoma, M., Nakazawa, K., \& Emori, H. 2000, ApJ, 537, 1013

Inaba, S., \& Ikoma, M. 2003, A\&Ap, 410, 711

Inaba, S., Tanaka, H., Nakazawa, K., Wetherill, G. W., \& Kokubo, E. 2001, Icarus, 149, 235

Johansen, A., Oishi, J. S., Mac Low, M.-M., Klahr, H., Henning, T., \& Youdin, A. 2007, Nature, 448, 1022

Kirsh, D. R., Duncan, M., Brasser, R., \& Levison, H. F. 2009, Icarus, 199, 197

Kobayashi, H., \& Tanaka, H. 2010, Icarus, 206, 735

Kobayashi, H., Tanaka, H., Krivov, A. V., \& Inaba, S. 2010 Icarus, 209,836

\section{REFERENCES}

Kobayashi, H., Tanaka, H., \& Krivov, A. V. 2011, ApJ, 738, 35

Kokubo, E., \& Ida, S. 1998, Icarus, 131, 171

Malhotra, R. 1995, AJ, 110, 420

Masset, F. S., D'Angelo, G., \& Kley, W. 2006, ApJ, 652, 730

Michikoshi, S., Kokubo, E., \& Inutsuka, S.-i. 2012, ApJ, 746, 35

Mizuno, H. 1980, Progress of Theoretical Physics, 64, 544

Morbidelli, A., \& Crida, A. 2007, Icarus, 191, 158

Ohtsuki, K., Stewart, G. R., \& Ida, S. 2002, Icarus, 155, 436

Okuzumi, S., \& Hirose, S. 2011, ApJ, 742, 65

Okuzumi, S., Tanaka, H., Kobayashi, H., \& Wada, K. 2012, ApJ, 752,106

Ormel, C. W., Dullemond, C. P., \& Spaans, M. 2010a, ApJ, 714, L103

Ormel, C. W., Dullemond, C. P., \& Spaans, M. 2010b, Icarus, 210,507

Ormel, C. W. \& Klahr, H. H. 2010, A\&A, 520, A43

Ormel, C. W., \& Kobayashi, H. 2012, ApJ, 747, 115

Paardekooper, S.-J., Baruteau, C., Crida, A., \& Kley, W. 2010, MNRAS, 401, 1950

Paardekooper, S.-J., Baruteau, C., \& Kley, W. 2011, MNRAS, 410, 293

Sano, T., Miyama, S. M., Umebayashi, T., \& Nakano, T. 2000, ApJ, 543, 486

Takeuchi, T., \& Ida, S. 2012, ApJ, 749, 89

Tanaka, H., Takeuchi, T., \& Ward, W. R. 2002, ApJ, 565, 1257

Tanigawa, T., \& Ikoma, M. 2007, ApJ, 667, 557

Walsh, K. J., Morbidelli, A., Raymond, S. N., O'Brien, D. P., \&

Mandell, A. M. 2011, Nature, 475, 206 